\title{
5G: ARCHITECTURE OVERVIEW AND DEPLOYMENTS SCENARIOS
}

\author{
Zaame Imane ${ }^{1}$, Tomader Mazri ${ }^{2}$, Elrhayour Amine ${ }^{3}$ \\ ${ }^{1}$ Ibn Tofail University, National School of Applied Sciences, KENITRA, MOROCCO \\ ${ }^{2}$ Ibn Tofail University, National School of Applied Sciences, KENITRA, MOROCCO \\ ${ }^{3}$ AFD.TECH, RABAT, MOROCCO \\ zaame.imane@gmail.com
}

KEY WORDS: 1G, 2G, 3G, 4G, 5G, SA, NSA, Architecture.

\begin{abstract}
:
Due to the exclusory, growth in the number of connected devices- predicted 50 billion connected devices in 2020 (Gary Davis, 2020) that demand internet access, an upgraded network is required. The fifth generation is the newest mobile network replacing the 4G technology. Deployed from 2020 in many countries, 5G will provide revolutionary innovations in mobile technologies that will reach various high-level goals to the costumers as well as Companies.To benefit from $5 \mathrm{G}$ network services, the whole word is growing up rapidly towards the $5 \mathrm{G}$ by developing a clear perspective to include the main challenges, opportunities and key technology components. In this paper, an attempt has been made to provide an overview about the evolution of mobile generations from $1 \mathrm{G}$ to $5 \mathrm{G}$ by comparing the challenges and features that have evolved from each generation, describe the various scenario of $5 \mathrm{G}$ deployment, and discuss the best choice Implementation of 5G NR.
\end{abstract}

\section{EVOLUTION OF 1G TO 5G TECHNOLOGY}

Cellular Mobile communication has generations as shown in the figure. Bellow a brief description of every generation:

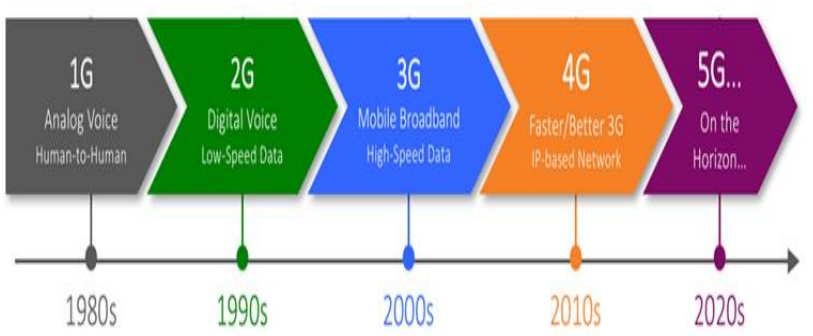

Figure 1. Block diagram of evolution of wireless network.

\subsection{G: Analogue Voice Technology}

The first generation wireless mobile communication system is an analogue technology developed in 1974 and completed in 1984 (Ms. Anju Uttam Gawas, 2015).

It was used to voice services based on an Advance Mobile Phone Service (AMPS) technology (Mohammad Meraj, 2015) AMPS system rely on frequency modulation radio system using Frequency Division Multiple Access (FDM) it allows only voice calls. Its speed up to $2.4 \mathrm{Kbps}$ (S.Venkata Krishna Kumar, 2014). there are few drawbacks in the $1 \mathrm{G}$ mobile system. First, it has a poor Voice Quality, poor Battery Life Large phone size. Secondly, No Security is required and Global Roaming Service was not possible. Finally, it does not have data service to convert the voice into digital signals.

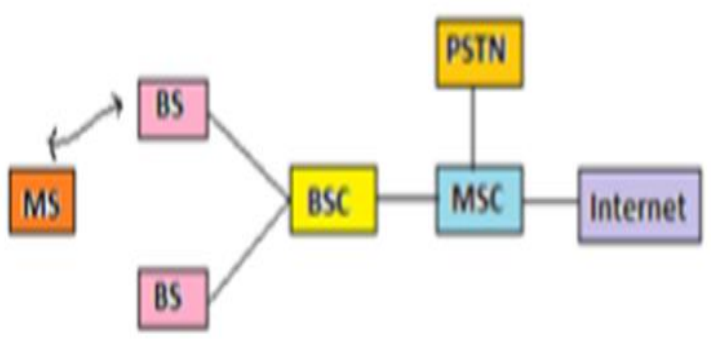

Figure 2. Architecture of Advance Mobile Phone Services (AMPS)

\subsection{G: Going Digital}

$2 \mathrm{G}$ denotes the second generation of mobile networks, which were the next stage in the development of mobile communication after 1G. 2G was started at 1980's and completed at 1990's, which were mainly for voice transmission with digital signals and the speed up to $64 \mathrm{kbps}$.

In 2G, two schemes such as Time Division Multiple Access (TDMA) and Code Division Multiple Access (CDMA) were used. Moreover, GSM (Global System for Mobile Communication) is the most widely used $2 \mathrm{G}$ mobile standard and it was the first one to support international roaming (Mohammad Meraj, 2015).

$2 \mathrm{G}$ was based on digital technology while $1 \mathrm{G}$ was analog. CDMA \& GSM appeared with the $2 \mathrm{G}$ network. It brought in other popular services we use today such as inter-nal roaming, MMS, SMS, conference calling, call hold, etc. 


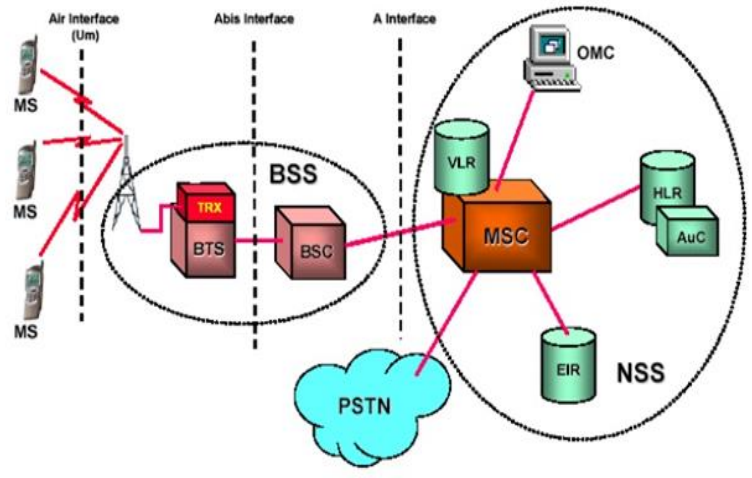

Figure 3. GSM System Architecture

The limitation of $2 \mathrm{G}$ reside in the fact that $2 \mathrm{G}$ requires strong digital signals to help mobile phones work. If there is no network coverage in any specific area, digital signals would weak. These systems are unable to handle complex data such as Videos.

\subsection{G: Driving data rates upwards with UMTS technology}

$3 \mathrm{G}$ is the third generation of mobile phone standards and technology, superseding $2 \mathrm{G}$, and preceding 4G. It was introduced in 2000; the goal of $3 \mathrm{G}$ systems was to offer increased data rates from $144 \mathrm{kbps}$ to $384 \mathrm{kbps}$ in wide coverage areas and $2 \mathrm{Mbps}$ in local coverage areas (Mohammad Meraj, 2015) and growth with minimum investment. High Bandwidth, Higher Speed, Price, Always Online Devices, Associated Costs, Power Requirements, Functions, Getting Information.

The 3G network is one of the main reasons for the popularity of smartphones; with $3 \mathrm{G}$, we were able to browse faster, check emails, streaming videos, social media sharing, etc.

3G mobile system was called as UMTS (Universal Mobile Telecommunication Sys-tem) in Europe, while CDMA2000 is the name of American $3 \mathrm{G}$ variant. In addition, the IMT2000 has accepted a new 3G standard from China, i.e. TD-SCDMA. WCDMA is the air-interface technology for UMTS (S. Ahmadi, 1999).

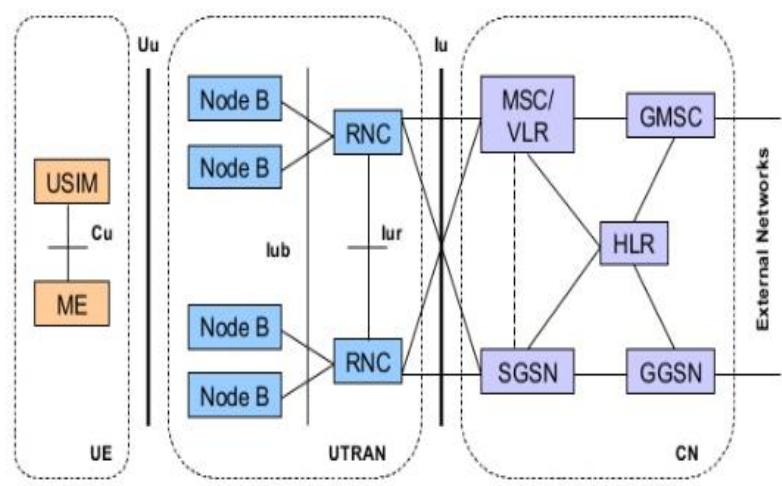

Figure 4. UMTS System Architecture

The limitations of $3 \mathrm{G}$ are expensive fees for $3 \mathrm{G}$ Licenses services; it was challenge to build the infrastructure for $3 \mathrm{G}$, High Bandwidth Requirement, and Expensive 3G Phones.

\subsection{G: All IP Network with LTE}

Work on 4G standardization started in 2004, just as 3G started to take-off. 4Grefers to Long Term Evolution (LTE) (Linge, N., and Sutton, A, 2014) and was all IP based network system.

The main goal of $4 \mathrm{G}$ technology is to provide high speed, high quality, high capacity, security and low cost services for voice and data services, multimedia and internet over IP. The reason for the transition to all IP is to have a common platform to all the technologies developed so far.

4G technology integrate different existing and future wireless technologies (e.g. OFDM, MC-CDMA, LAS-CDMA and Network-LMDS) to provide freedom of movement and uninterrupted roaming from one technology to another technology developed so far (Mohammad Meraj, 2015).

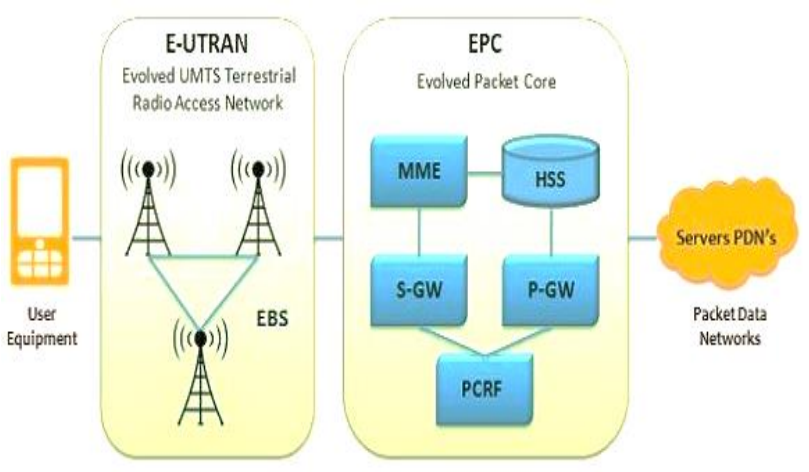

Figure 5. LTE System Architecture

The basic difference between $3 \mathrm{G}$ and $4 \mathrm{G}$ is in data transfer and signal quality. 3G 4G Data Transfer Rate $3.1 \mathrm{MB} / \mathrm{sec} 100$ $\mathrm{MB} / \mathrm{sec}$ Internet Services Broadband Ultra Broadband Low High Bandwidth 5-20 MHz 100MHz Frequency 1.6-2 GHz 2-8 $\mathrm{GHz}$ Download and upload 5.8 Mbps 14 Mbps Technology Mobile - TV Resolution. Moreover, there is no Circuit Switched Domain in $4 \mathrm{G}$

\section{$1.55 G$}

Fifth Generation $(5 \mathrm{G})$ of mobile communication, started from late 2010, aims to provide a better level of connectivity and coverage, complete wireless communication with almost no limitations and enhanced quality of service (QoS). It is highly supportable to WWWW (Wireless World Wide Web) (Jonathan Rodriguez, 2015).

The $5 \mathrm{G}$ NR is a new aerial interface developed for $5 \mathrm{G}$. This is the radio frequency part of the circuit between the mobile terminal and the base station.

5G will initially be available through improvements in LTE, LTE-Advanced and LTE Pro technologies. However, it will soon be followed by a major step with the introduction of a new radio interface (Ericsson, 2019). 


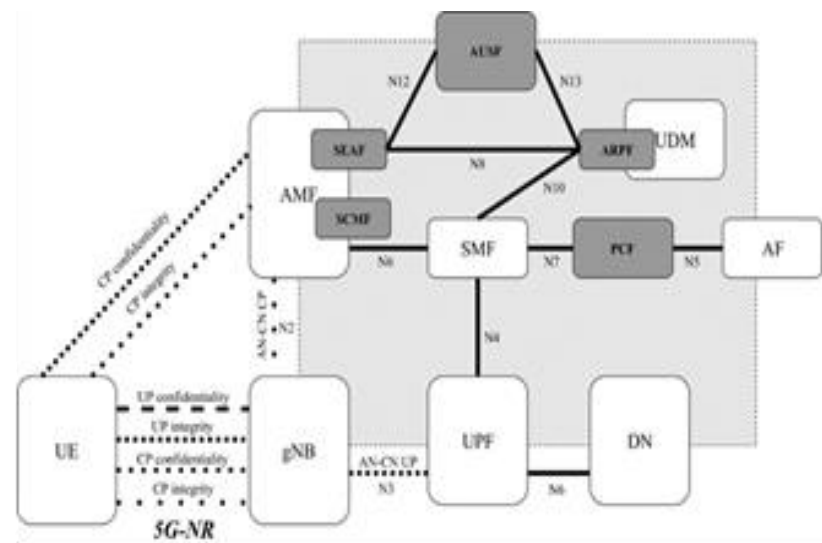

Figure 6. 5G System Architecture

5G NR core will include three fundamental elements described in the figure below:

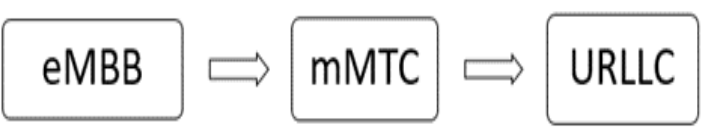

Figure 7. The top three cases of 5G uses

- Enhanced Mobile Broadband (eMBB): Including data speeds of several gigabits per second (Gbit/s) for applications such as virtual reality and the ability to support significant growth in data traffic.

- Ultra-reliable and Low-latency Communications (uRLLC) or Mission-Critical Control: for latency-sensitive services requiring extremely high reliability, availability and safety, such as autonomous driving.

- Massive Machine Type Communications (mMTC) or Massive IoT: Offering the ability to support a large number of low-cost IoT connections with a very long battery life and wide coverage, including inside buildings (Erik Guttman).

The RAN based on the New Radio (NR) standard will provide high levels of capacity and peak data rates along with low latency. These networks can provide $10 \mathrm{Mbits} / \mathrm{s}$ to 10 of 100 of users at the same time.

\section{DEPLOYMENTS SCENARIOS FOR 5G NR}

3GPP introduce the 5G standards in Release 15 to provide guidelines for $5 \mathrm{G}$ networks. These standards aim to provide massive throughput and low latency to the end user.

There are different phases under which 5G NR (New Radio) will be deployed as per 3GPP specifications published in the December 2017 (Gabriel Brown). phase 1 (Rel-15, 06.18 stage 3 freeze) and phase 2 (Rel-16, 12.19 stage 3 freeze).

$5 \mathrm{G}$ can be deployed in five different deployment options, where SA (standalone) options consist of only one generation of radio access technology and NSA options consist of four generations of radio access technologies (4G LTE and 5G).

3GPP has defined two solutions for $5 \mathrm{G}$ networks as follows:

\subsection{G Standalone (SA):}

The 5G Standalone architecture SA will depend on 5G New Radio (5G NR) and 5G Core Network (5GC). New capabilities like Network Slicing using E2E, CUPS, Virtualization, MultiGbps support, and Ultra low latency will be inherently built into the 5G SA Packet Core architecture (Zetao Xu, Yang Zhang, Ao Shen, Bao Guo, Yuehua Han and Yi Liu, 2019).

The salient features of SA implementation are:

- 5G will be used for both C-Plane and U-Plane.

- All radio control parameters will be exchanged through 5G.

- Paging channels will be monitored by UE on 5G.

\subsection{G Non-Standalone (NSA):}

The Non-Standalone deployment based on the already existing LTE radio access and core network (EPC) as an anchor for mobility management and coverage to add the $5 \mathrm{G}$ carrier. This solution will be more cost-efficient and can be provided in a shorter time.

NSA mode is based on EUTRA-NR Dual connectivity (ENDC) where $5 \mathrm{G}$ radio will work with $4 \mathrm{G}$-core network and will give hotspot coverage along with the existing $4 \mathrm{G}$ network.

In NSA, 5G will only focus on U-Plane; all the C-plane messages like call origination/termination, location registration, etc. will be handled by the LTE eNB and EPC (Zetao Xu, Yang Zhang, Ao Shen, Bao Guo, Yuehua Han and Yi Liu, 2019).

\subsection{Option 1: SA LTE under EPC}

Option 1 represents current 4G (LTE) deployments. It is also called Standalone LTE or EPC connected system. At present, most operators may have already deployed this option.

The SA option is a simple solution for operators to manage and can be deployed as an independent network using the Handover between $4 \mathrm{G}$ and $5 \mathrm{G}$ for service continuity.

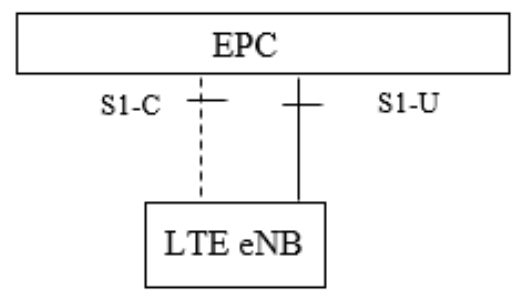

Figure 8. Option 1

\subsection{Option2: SA NR under 5GC}

This deployment scenario will be particularly beneficial in areas where there is no LTE system and where the operator wants to deploy a full-fledged 5G NR access system without 4G interconnection. 
This option allows the operator to implement all types of 5G use cases such as eMBB, mMTC and URLLC.

A prerequisite for this option is that the operator should have multiple spectra so that they can provide ubiquitous 5G coverage and enjoy all the benefits.

Standalone option 2 is where radio access network consists of only gNBs and connects to $5 \mathrm{G}$ Core, and the $5 \mathrm{GC}$ interworks with EPC. SA option 2 has no impact on LTE radio and can fully support all $5 \mathrm{G}$ use cases.

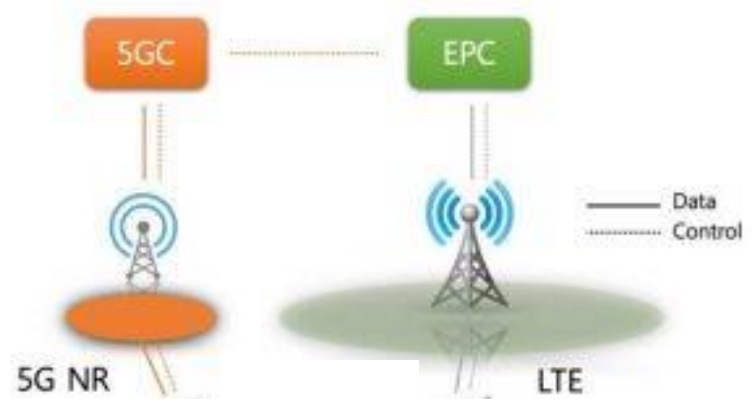

Figure 9. High-level 5GC SA Option 2 interworks with EPC

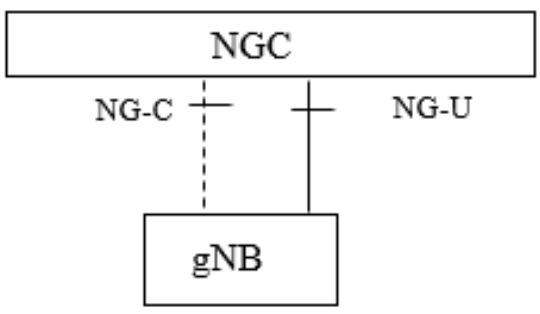

Figure 10. Option 2

In SA NR, the gNB connects directly to the $5 \mathrm{G}$ core. The SA NR system has been defined in the $5 \mathrm{G}$ specifications, for $3 \mathrm{GPP}$ Rel-15.

The benefits of this option are:

- The operator can fully leverage 5G E2E (End-to-end) capabilities.

- All specifications have been standardized to 3GPP Rel-15 (September-2018)

- The user plan and the control plan are both managed by $5 \mathrm{G}$ network.

- The operator can deploy Inter-RAT mobility between LTE/EPC and NR / 5GC.

- The operator can choose between Voice fallback on VoLTE, or IMS Voice on NR (VoNR).

The drawbacks of this approach are:

- Direct deployment of 5G requires more investment.

- The operator cannot take advantage of existing deployments of the existing network (LTE) in the short term.
This phase promises the greatest potential for future evolution and growth, with many new capabilities introduced in 5GC. The devices will also be able to handle many new services.

\subsection{Option 3: NSA LTE and NR under EPC}

Option 3 is a NSA scenario where the network still use LTE with NR radio access, but using only LTE's EPC core to deliver control signals. In this option, LTE is used as the anchor of the control plan for NR, LTE and NR are used for user data traffic (User Plan).

It could also be called non-Standalone (NSA) NR in Evolved Packet System.

\subsubsection{Option 3}

In option 3, ENB is the master that send and receive all Uplink and Downlink data, also decide which part of the data would send to the $4 \mathrm{G} / 5 \mathrm{G}$ station using X2 interface. Therefore, 5G gNB does not have any direct communication with LTE core network.

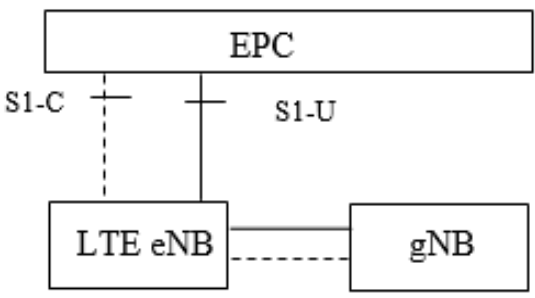

Figure 11. Option 3

Hardware upgrade is probably required, because they would be more additional traffic to be handled.

\subsubsection{Option 3a}

This option allowed to $5 \mathrm{G}$ gNB and LTE ENB to communicate directly with $4 \mathrm{G}$-core network without having communication between them over the $\mathrm{X} 2$ interface. There is only control plane traffic in the $\mathrm{X} 2$ interface. So the $\mathrm{X} 2$ traffic is very small.

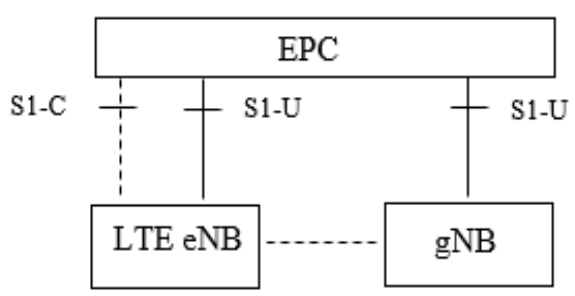

Figure 12. Option 3a

This means that there can be no load sharing of data over a single bearer over $4 \mathrm{G}$ and $5 \mathrm{G}$. That means, for example, that the $4 \mathrm{G}$ part only handles the VoLTE voice traffic for a user while his Internet traffic is only handled by the $5 \mathrm{G}$ part of the base station. Say that in most deployment scenarios, this is not really an option if mobile devices move in and out of $5 \mathrm{G}$ network coverage continuously. 


\subsubsection{Option 3x}

$3 \mathrm{x}$ is a combination of 3 and $3 \mathrm{a}$, so both $\mathrm{S} 1$ and $\mathrm{X} 2$ interfaces are available for User plan so the traffic can be split based on the backhaul capacity of S1-U.

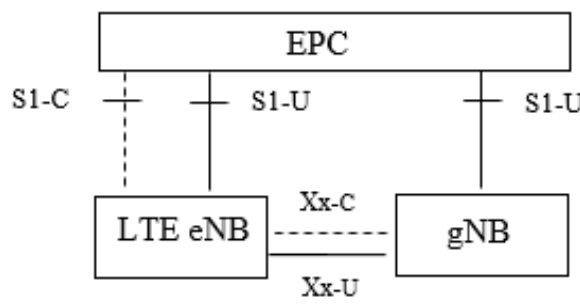

Figure 13. Option 3x

- Option 3x provides robust coverage in higher frequencies and aggregated peak bit rate of LTE and 5G for lower frequencies.

- Option 3x also provides near zero interrupt time LTE-5G mobility.

- Option-3x provides allows voice in LTE without using RAT fallback.

- This configuration can be used in scenarios where LTE coverage reach is superior to that of NR and thus leverages EPC.

\subsection{Option4: NSA NR and LTE under 5GC}

With option 4, the gNB act as the master node and eNB is the secondary node, so the NR RAN will be in charge of C-Plan signalization.

\subsubsection{Option 4}

In option 4, there is no direct connectivity between ng-eNB and 5GC.All the information follows via Xn interface that should be newly designed.

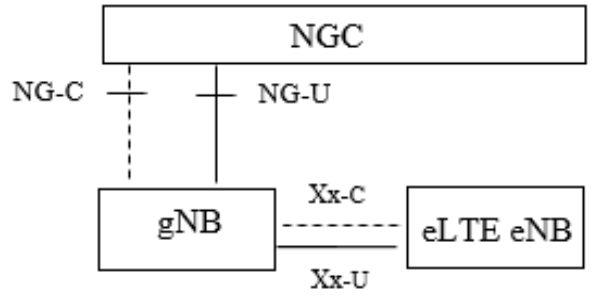

Figure 14. Option 4

\subsubsection{Option 4a}

In option $4 \mathrm{a}$, there is no $\mathrm{Xn}$ interface between gn-eNB and $\mathrm{gNB}, \mathrm{gn}-\mathrm{eNB}$ is connected to $5 \mathrm{GC}$ via $\mathrm{NG}-\mathrm{U}$ interface.

This option also option 4 required an upgrade (release 15) of eLTE and a strong $5 \mathrm{G}$ coverage.

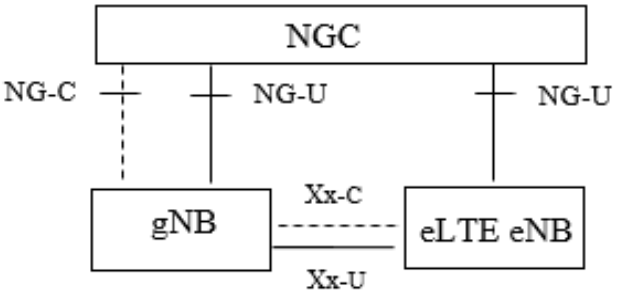

Figure 15. Option 4a

\subsection{Option5: SA LTE under 5GC}

In this option, the network made the transition to the NGC, but continued the use of LTE access. LTE, in this case, is an advanced LTE RAN that includes new signage.

Given that most of the benefits of $5 \mathrm{G}$ will come from migrating to a new radio, this option seems unlikely.

Stand Alone (SA) Evolved E-UTRA (LTE) in 5G Standalone (5GS: This deployment scenario is particularly suited to areas that do not have an inherited LTE system and advanced EUTRAN access systems are deployed. In this deployment, the ng-eNB is connected to $5 \mathrm{GC}$.

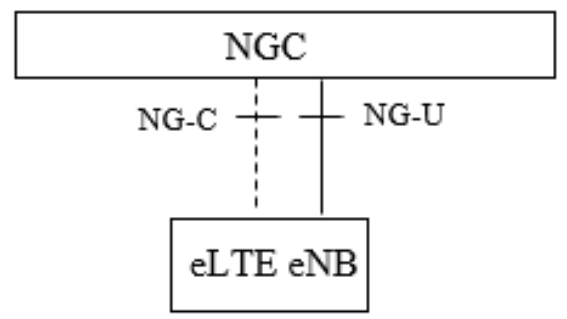

Figure 16. Option 5

One important point to consider here is that it not all of these intermediate options can be practically implemented.

\subsection{Option7: NSA LTE and NR under 5GC}

The last NSA option is option 7 LTE assisted NR ,instead of using EPC core in option 3, we use 5G core using the $\mathrm{Ng}$ interfaces rather than $\mathrm{Xx}$ interfaces, and to handle those interfaces the eNodeB must be upgraded to the next generation eNB(ng-eNB) (3GPP release 15 ).

We have three sub-options:

\subsubsection{Option 7}

In option 7 there is no interfaces between gNB and $5 \mathrm{GC}$ the data flows via $\mathrm{Xn}$ inter-faces. 


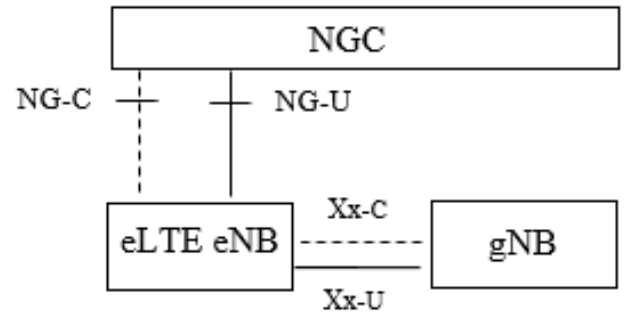

Figure 17. Option 7

\subsubsection{Option 7a}

The difference between 7 and $7 \mathrm{a}$ is that U-plane data is no longer sended via $\mathrm{Xn}$, but via NG-U, so here appeared the characteristic of NSA option when they use ng-LTE as an anchor to have a connectivity with 5GC.

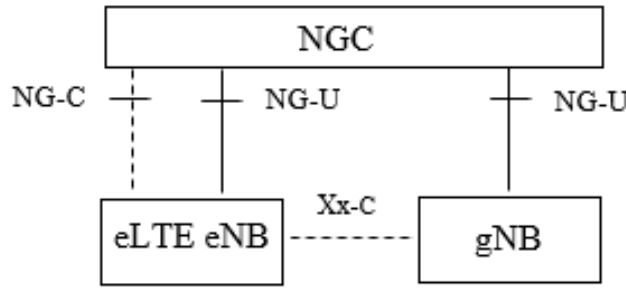

Figure 18. Option 7a

\section{DISCUSSION}

The objective of telecom companies is to be in tune with the rapid growth of technology markedly $5 \mathrm{G}$ and have the fastest or largest 5G networks. In addition, countries are competing to be the first to deploy fully functional, nationwide $5 \mathrm{G}$, with the fastest smoothest and most efficient way possible.

As shown in this paper, there are many scenarios to deploy $5 \mathrm{G}$, but with the pressure to improve performance and handle the increase demand of customers.

Option $3 \mathrm{x}$ is the best choice to deploy $5 \mathrm{G}$ as soon as possible without waiting a fully break up with LTE.

Therefore, in option 3x, operators can take full advantages of the existent LTE legacy using only EPC to route command signals.

Adding that with Dual connectivity, option 3x will give to the costumer's opportunity to benefit from the eMBB, such as a higher data rate in Downlink NR, also an interesting coverage in the Uplink LTE.

5G NSA Option 3x will not introduce any changes to the existing roaming architecture and procedures. It is up to the VPLMN (Visited Public Land Mobile Network) operator to allow inbound roamers to use 5G NSA Option $3 \mathrm{x}$ or to only allow SA/LTE (Option 1).

In addition, option $3 \mathrm{x}$ is the shortest and lowest way to integrate 5G SA system and keeping the connectivity, interoperability with all generation specially the incoming generation $6 \mathrm{G}$ and $7 \mathrm{G}$.

\section{CONCLUSION}

This paper introduces five network architectures of 5G non-standalone and standalone networking explain what is the most likely option that is going to be adopted by operators across the world and focuses on the option $3 \mathrm{x}$ in $5 \mathrm{G}$ non-standalone networking as a possible first step in the migration towards 5G-SA. Therefore, the operators can either start with Option 3x, upgrade to Option 7x and then migrate to Option 2 (SA), or go directly from Option $3 x$ to Option 2.

\section{REFERENCES}

Gary Davis, 2020: Life with 50 billion connected devices. In: IEEE, F., McAfee, Inc. CONFERENCE 2018, ICCE, vol. 9781-5386-3025-9/18/\$31.00 @2018 IEEE, pp. 1-1. Las Vegas, USA (2018).

Ms. Anju Uttam Gawas, International Journal on Recent and Innovation Trends in Compu-ting and Communication 31303133 (2015).

Mohammad Meraj ud in Mir et al, / (IJCSIT) International Journal of Computer Science and Information Technologies, Vol. 6 (3), 2015, 2545-2551).

S.Venkata Krishna Kumar "A Study of Wireless Mobile Technology"International Journal of Advanced research in computer science and software engineering, vol.4, issue, Jan 2014, ISSN: 2277-128x

S. Ahmadi, in Academic Press Library in Mobile and Wireless Communications, 2016 Au-thor, F., Author, S., Author, T.: Book title. 2nd edn. Publisher, Location (1999).

Linge, N., and Sutton, A. The road to 4G. The Journal of the Institute of Telecommunica-tions Professionals,Vol 8(1), Mar 2014.

Jonathan Rodriguez. Fundamentals of 5G Mobile Networks. This edition first published 2015@ 2015 John Wiley \& Sons, Ltd.

Ericsson white paper GFMC-19:000148 March 2019.

Erik Guttman 3GPP TSG SA Chairman Samsung Electronics R\&D Institute, UK.

Gabriel Brown, Service-oriented 5G Core Networks, Huawei Technologies Co.Ltd (2017).

Zetao Xu, Yang Zhang, Ao Shen, Bao Guo, Yuehua Han and Yi Liu, Initial Analysis of the Cell Selection Progress in SA of 5G NR,In: 5th International Conference on Signal and In-formation Processing, Networking and Computers (ICSINC) Volume 550,DOI: 10.1007/978-981-13-7123-3_58Henan, China (2019).

A Slalmi, H Kharraz, R Saadane, C Hasna, A Chehri, G Jeon 2019 15th International Conference on Signal-Image Technology \& Internet, 2019. 